\title{
Star Formation: Chemistry as a Probe of Embedded Protostars
}

\section{Ruud Visser*}

Department of Astronomy, University of Michigan, 500 Church Street, Ann Arbor, MI 48109-1042, USA

E-mail: visserreumich.edu

\begin{abstract}
The embedded phase of star formation is the crucial phase where most of the stellar mass is assembled. Velocity-resolved spectra reveal an infalling envelope, bipolar outflows, and perhaps an infant circumstellar disk - all locked together in a cosmic dance of gravitational collapse and magnetic winds. Densities and temperatures change by orders of magnitude as the protostar evolves, driving a chemistry as exotic as it is fascinating. I will review two examples of how to exploit chemistry and molecular spectroscopy to study the physics of low-mass star formation: energetic feedback and episodic accretion.
\end{abstract}

Frank N. Bash Symposium 2013: New Horizons in Astronomy (BASH 2013)

October 6-8, 2013

Austin, Texas

${ }^{*}$ Speaker. 


\section{Introduction}

For all of human history, we have wondered about the origins of the Earth, the Sun, and the rest of the solar system. The notion that stars form from the gravitational collapse of a molecular cloud core traces back to the "nebular hypothesis" developed by Swedenborg (1734), Kant (1755), and Laplace (1796). Since then, many generations of astronomers have filled in the details of what such cloud cores look like, how they collapse, and how this results in a planetary system (Cameron 1988). Others have studied the differences between low-mass and high-mass stars, between stars born in isolation or in clusters, and between star formation in the Milky Way or other galaxies (Kennicutt \& Evans 2012).

The state of affairs in the field of star formation has been reviewed periodically, in part through the Protostars and Planets series of conferences. The most recent edition, PPVI, took place in July 2013 in Heidelberg, Germany and featured chapters on molecular clouds (Dobbs et al. 2014; André et al. 2014), embedded protostars (Li et al. 2014; Dunham et al. 2014), chemical evolution (van Dishoeck et al. 2014; Ceccarelli et al. 2014), and much more. Earlier reviews on various aspects of star formation were written by, e.g., Shu et al. (1987), van Dishoeck et al. (1993), van Dishoeck \& Blake (1998), Lada (1999), André et al. (2000), Klein et al. (2007), White et al. (2007), and McKee \& Ostriker (2007).

The process of low-mass star formation is usually split into four to six steps, as illustrated in Fig. 1. The first step (panel a) is a molecular cloud of typically a few pc in size with a mean density of about $100 \mathrm{~cm}^{-3}$ and a total mass of a few $1000 M_{\odot}$ (Bergin \& Tafalla 2007). The cloud contains denser clumps of a few $100 M_{\odot}$ each, which in turn contain individual dense cores $\left(10^{4}-10^{5} \mathrm{~cm}^{-3}\right)$ of a few $M_{\odot}$ in mass and about $0.1 \mathrm{pc}$ in size. In many star-forming regions, the clumps and cores line up in filaments (André et al. 2014).

Star formation properly starts when a dense core loses press ure support and begins to undergo gravitational collapse (Fig. 1b). Due to the core's rotation, material falling in from the outer parts gets deflected towards the gravitational midplane. The young star soon launches a bipolar jet that carries away some of the core's angular momentum. The jet carves out a bipolar cavity from the infalling envelope and drives an outflow into the surrounding molecular cloud (Fig. 2; Bally et al. 2007; Ray et al. 2007; Arce et al. 2007; Shang et al. 2007; Frank et al. 2014). Initially, when the young star is still deeply embedded in its natal core, it is called a Class 0 or Stage 0 protostar (André et al. 1993; see Robitaille et al. 2006 for the distinction between observational classes and evolutionary stages). The transition to Stage I (Fig. 1c) occurs when the star has accreted enough matter that its mass exceeds that of the remnant circumstellar reservoir (Lada 1999; Robitaille et al. 2006). The median lifetimes for Stage 0 and I protostars - together called the embedded stage are 0.10 and 0.44 Myr (Evans et al. 2009).

After the envelope is cleared away, we are left with a pre-main-sequence T Tauri star surrounded by a circumstellar disk of gas and dust (Fig. 1d; Bertout 1989; Dullemond et al. 2007). Although disks of one form or another have now been detected in Stage 0 and I sources (e.g., Jørgensen et al. 2007; Takakuwa et al. 2012; Tobin et al. 2012; Murillo et al. 2013), it remains unclear how and when disks first form and how big they grow in the embedded phase (Li et al. 2014). In $\mathrm{T}$ Tauri stars, disks can be anywhere from less than $10 \mathrm{AU}$ to a few $100 \mathrm{AU}$ in radius and their mass can be as high as $0.1 M_{\odot}$ (Andrews \& Williams 2007). They last for a few Myr, during which 

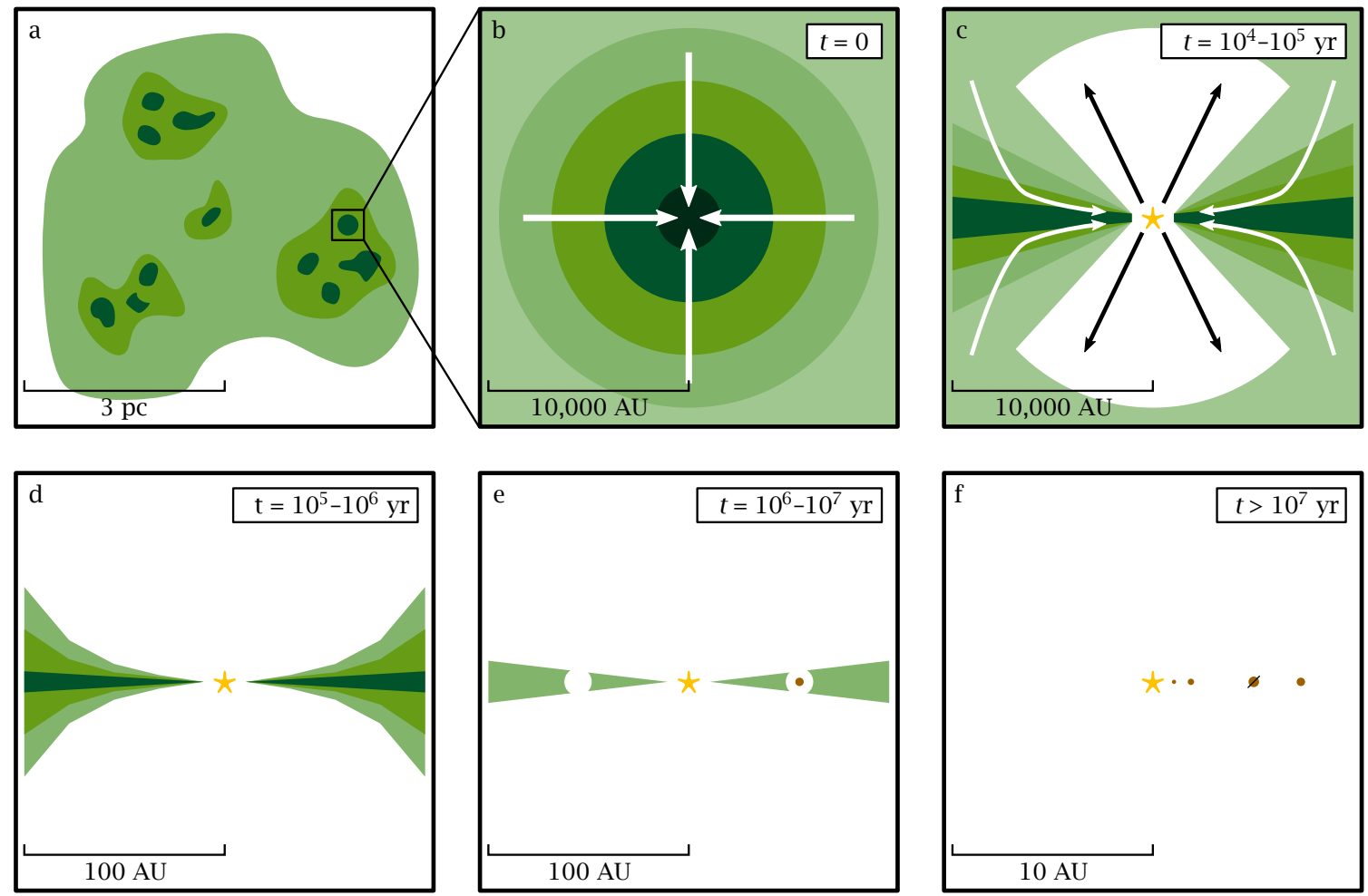

Figure 1: Schematic view of isolated low-mass star formation, based on Shu et al. (1987). Darker colors represent higher densities. The typical sizes and timescales for each step are indicated.

the dust settles towards the midplane and grows first to larger grains, then to rocks and boulders, and ultimately to planets (Johansen et al. 2014; Raymond et al. 2014; Helled et al. 2014; Benz et al. 2014). Meanwhile, the gas in the disk gradually gets dispersed into the interstellar medium (Alexander et al. 2014) or accreted by giant planets. The combined effects of planet formation and gas dispersal can leave gaps and holes, producing a so-called transitional disk (Fig. 1e; Espaillat et al. 2014). After the gas is cleared away, the system turns into a debris disk in which rocky planets can continue to grow (Matthews et al. 2014). This phase can last for another 10-100 Myr before we end up with a main-sequence star and a mature planetary system (Fig. 1f).

The current review covers two topics of recent interest in the embedded phase of star formation: energetic feedback (Section 2) and episodic accretion (Section 3). Both parts discuss in particular the use of chemistry and molecular spectroscopy as a probe of the the underlying physical processes.

\section{Energetic feedback}

Star formation is a violent process. As the cloud core collapses and material accretes onto the young star, the gravitational potential energy of the infalling gas is released back into the protostellar environment (McKee \& Ostriker 2007). Nuclear fusion within the star forms an additional source of energy output; for low-mass stars, this begins at about $0.1 \mathrm{Myr}$ after the onset of collapse (Stahler 1983; Myers et al. 1998). 


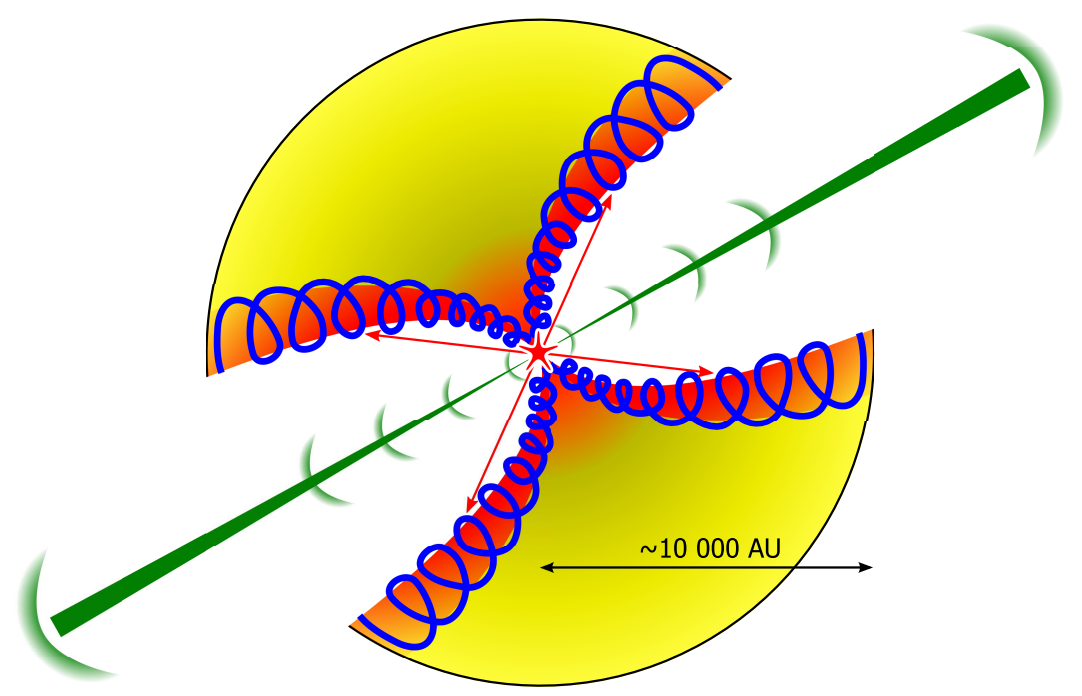

Figure 2: Schematic representation of a low-mass embedded protostar from Visser et al. (2012). The gas temperature in the infalling envelope ranges from $10 \mathrm{~K}$ at the outer edge (yellow) to a few $100 \mathrm{~K}$ close to the star (red). The protostellar UV field illuminates the gas in the walls of the bipolar outflow cavity and heats it to a few $100 \mathrm{~K}$ even at large distances (red). Shocks driven by the jet (green) or the stellar wind can heat the gas in the cavity walls to a few $1000 \mathrm{~K}$.

Feedback is important in regulating the overall star-formation efficiency, i.e., the fraction of total cloud mass that actually makes it into stars (Shu et al. 1987, 2004; Evans et al. 2009). On galactic scales, feedback from massive stars shapes the interstellar medium through ionizing radiation and stellar winds (Chu 2008; Arthur 2008; Dopita 2008; Oey et al. 2008). Low-mass protostars exert much the same type of feedback on their environment, but on much smaller scales: they affect the physics and chemistry of the circumstellar disk and envelope through processes like photoelectric heating, ionization, and shock compression (Visser et al. 2012). In numerical simulations of low-mass star formation, the higher temperatures due to radiative feedback enhance the stability of the disk (reducing fragmentation) and produce a better fit to the initial mass function (Offner et al. 2010; Bate 2012).

The warm and hot gas (from 100 to a few $1000 \mathrm{~K}$ ) in low-mass protostars emits mainly at mid- to far-infrared wavelengths. Every escaping photon carries away a quantum of energy; the dominant "cooling lines" are pure rotational transitions of $\mathrm{H}_{2}, \mathrm{CO}, \mathrm{H}_{2} \mathrm{O}$, and $\mathrm{OH}$ (Giannini et al. 2001; van Dishoeck 2004; Neufeld et al. 2009; Karska et al. 2013). Over the past decade, the Spitzer Space Telescope (5-37 $\mu \mathrm{m}$; Werner et al. 2004) and the Herschel Space Observatory (55$670 \mu \mathrm{m}$; Pilbratt et al. 2010) have provided a wealth of data, building on earlier pioneering work with the Infrared Space Observatory (ISO; 2.5-200 $\mu \mathrm{m}$; Kessler et al. 1996). For example, Spitzer covered the lowest eight rotational lines of $\mathrm{H}_{2}$ with upper-level energies up to $7200 \mathrm{~K}$ (Neufeld et al. 2009), along with many lines of $\mathrm{H}_{2} \mathrm{O}$ (700-3000 K) and some of $\mathrm{OH}$ (400-3000 K; Watson et al. 2007). Herschel filled in the lower-excitation range of $\mathrm{H}_{2} \mathrm{O}$ and $\mathrm{OH}$, and covered the $\mathrm{CO}$ rotational ladder up to the $J=49-48$ transition with $E_{\text {up }}=6700 \mathrm{~K}$ (Herczeg et al. 2012).

The cooling lines observed with Spitzer and Herschel can originate in various parts of the protostar (Fig. 2). Quantifying and disentangling the contributions from each physical component 


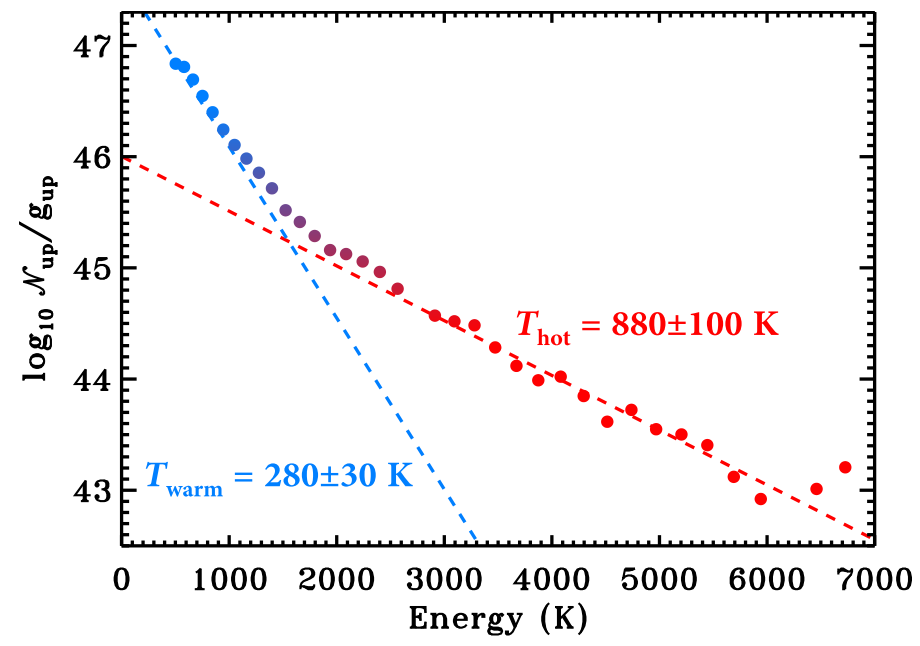

Figure 3: Rotational diagram for CO in the Class 0 protostar NGC1333 IRAS4B, adapted from Herczeg et al. (2012). The vertical axis plots the total number of detected molecules for each $J$ level divided by the degeneracy of that level.

has been a major challenge, not in the least because most of the observations are spatially and spectrally unresolved. ${ }^{1}$ I will discuss the CO rotational lines in more detail as an example of both the opportunities and the limitations from the Herschel observations.

Rotationally excited $\mathrm{CO}$ is ubiquitous in low-mass embedded protostars. Early observations with ground-based sub-millimeter telescopes and with ISO measured line intensities from $J=6-5$ up to 21-20 that far exceed any reasonable predictions for just an infalling envelope heated by the protostellar luminosity (Spaans et al. 1995; Hogerheijde et al. 1998; Giannini et al. 2001; Nisini et al. 2002). Two alternative heating mechanisms were suggested: ultraviolet photons and shocks.

The PACS spectrometer on Herschel had access to nearly all CO lines from $J=13-12$ up to 49-48 (Poglitsch et al. 2010). The three key programs DIGIT, HOPS, and WISH combined to observe the CO ladder in more than 50 low-mass embedded protostars (van Kempen et al. 2010a,b; Visser et al. 2012; Herczeg et al. 2012; Goicoechea et al. 2012; Manoj et al. 2013; Karska et al. 2013; Green et al. 2013; Dionatos et al. 2013; Lee et al. 2013; Lindberg et al. 2014). A common way to plot these data is in a rotational diagram, with the upper-level energies for the observed lines on the horizontal axis and the population of each rotational level on the vertical axis (Goldsmith \& Langer 1999). For optically thin emission and gas in local thermodynamic equilibrium (LTE), the points in such a diagram fall on a straight line. The slope of this line is then the inverse of the gas temperature.

For sources with a sufficient number of detected lines, the rotational points generally do not fall on a straight line and instead require two temperature components for a good fit (Fig. 3; Herczeg et al. 2012; Manoj et al. 2013; Karska et al. 2013; Green et al. 2013). The breakpoint falls at $E_{\text {up }} \approx 1800 \mathrm{~K}$ in all sources, between the 25-24 and 26-25 transitions. Although the full source sample spans more than two orders of magnitude in bolometric luminosity, the two temperature

\footnotetext{
${ }^{1}$ Towards the short end of their respective wavelength ranges, Spitzer and Herschel had a resolution of 500-2000 AU in nearby star-forming regions. The notable exception to the spectrally unresolved observations are the data from the heterodyne spectrometer HIFI on Herschel (de Graauw et al. 2010).
} 
components are remarkably constant: $320 \pm 50 \mathrm{~K}$ (“warm") and $850 \pm 150 \mathrm{~K}$ ("hot"). ${ }^{2}$ What do these temperatures mean and why do they show so little variation across more than 50 protostars?

One possible explanation is that the two temperature fits reflect two different reservoirs of $\mathrm{CO}$ gas. Detailed radiative transfer models constructed for NGC1333 IRAS2A, HH46, and DK Cha were able to reproduced the CO intensities with a combination of UV- and shock-heated gas (van Kempen et al. 2010b; Visser et al. 2012). Based in part on spectrally resolved CO 16-15 data from Herschel-HIFI, Kristensen et al. (2013) concluded that the "hot" component originates in dense gas $\left(>10^{6} \mathrm{~cm}^{-3}\right.$ ) within $100 \mathrm{AU}$ of the central star. The UV-heated gas appears to be more spread out, up to a few $1000 \mathrm{AU}$, but still has to have similarly high densities to maintain LTE. Therein lies the weakness of this scenario: why would a sample of more than 50 protostars, spanning more than two orders of magnitude in $L_{\mathrm{bol}}$, always have UV-heated gas of about $300 \mathrm{~K}$ ?

An alternative explanation is that the $\mathrm{CO}$ emission originates in subthermally excited gas (nonLTE) with a density of less than $10^{6} \mathrm{~cm}^{-3}$ (Neufeld 2012; Manoj et al. 2013). The gas can either be uniformly hot $(>2000 \mathrm{~K}$ ) or feature a continuous range of temperatures from a few 10 to a few 1000 K. Such conditions are consistent with shocks along the outflow or the cavity walls at several 100 to $1000 \mathrm{AU}$ from the central star. The advantage of the non-LTE solution is that it does not require a constant gas temperature across the source sample; instead, a fairly wide range of temperatures can all produce the same rotational excitation conditions. However, it is unclear whether the column density of hot, low-density gas is sufficient to match the observed line intensities. Invoking shocked gas as the sole origin of the high- $J \mathrm{CO}$ emission is also inconsistent with the narrow line profiles seen in velocity-resolved spectra of CO 6-5, 10-9, and 16-15, indicative of a reservoir of quiescent hot gas (van Kempen et al. 2009; Yıldız et al. 2012, 2013; Kristensen et al. in prep.).

\section{Episodic accretion}

\subsection{Solving the luminosity problem}

In the simplest theories of star formation, there is a constant flow of matter from the collapsing envelope onto the star and the stellar mass grows at a constant rate $\dot{M} \equiv \mathrm{d} M_{*} / \mathrm{d} t$ (Shu 1977). The stellar luminosity scales with the mass accretion rate and with the ratio of the stellar mass over the stellar radius (Adams \& Shu 1985):

$$
L_{*} \approx \frac{G M_{*} \dot{M}}{R_{*}} .
$$

The constant flow from these simple theories continues until the infalling envelope is depleted at the end of the embedded phase. T Tauri stars show some ongoing accretion from the disk onto the star, but at a rate that is two or three orders of magnitude slower than in Class 0 and I sources.

If the accretion rate is indeed constant throughout the embedded phase, a sample of low-mass protostars at the same evolutionary state should have roughly the same luminosity. Furthermore, embedded protostars should be more luminous than T Tauri stars. However, surveys with the Infrared Astronomical Satellite (IRAS), Spitzer, and Herschel have all shown that neither prediction holds true (Kenyon et al. 1990, 1994; Kenyon \& Hartmann 1995; Dunham et al. 2008; Enoch et al. 2009; Evans et al. 2009; Fischer et al. 2013). For example, Fig. 4 plots the bolometric luminosities

\footnotetext{
2 The missing "cold" component dominates the CO lines up to about $J=10-9$ (Y1ldiz et al. 2013).
} 


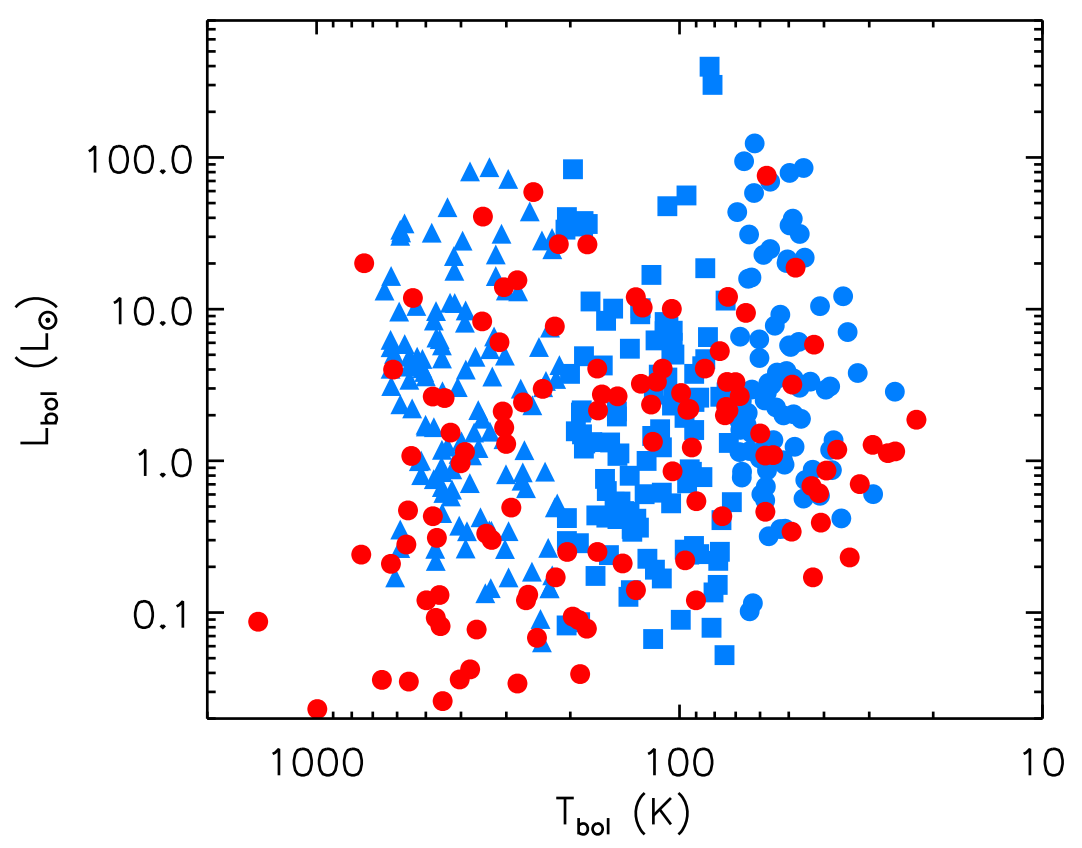

Figure 4: Bolometric temperatures and luminosities for the embedded protostars from Evans et al. (2009, red) and Fischer et al. (2013, blue). The former are mixed Class 0 and I; the latter are split into Class 0 (circles), early Class I (squares), and late Class I (triangles).

versus the bolometric temperatures ${ }^{3}$ for several hundred Class 0 and I sources observed with Spitzer and Herschel. The luminosities span more than three orders of magnitude at any given bolometric temperature (i.e., evolutionary state), which cannot be explained by a constant mass accretion rate onto the star.

Two possible solutions have been proposed for this "luminosity problem": an accretion rate that decreases with time, or a low accretion rate for most of the time interspersed by short bursts of high accretion (Kenyon et al. 1990). Accretion bursts are known to occur in T Tauri stars (FUor and EXor events; Herbig 1977; Hartmann \& Kenyon 1996; Audard et al. 2014). Similar bursts in embedded sources should be detectable at mid-IR, far-IR, and sub-mm wavelengths (Johnstone et al. 2013). A survey of 4000 protostars with a five-year baseline identified 1-4 burst candidates (Scholz et al. 2013). The presence of periodic shocks along protostellar jets also suggests that burst of some sort probably occur (Reipurth 1989; Raga et al. 2002). Radiative transfer calculations find better agreement with the $L_{\mathrm{bol}}$ and $T_{\mathrm{bol}}$ observations from Fig. 4 for scenarios with accretion bursts than they do for a steadily declining accretion rate (Myers et al. 1998; Offner \& McKee 2011; Dunham \& Vorobyov 2012). The "episodic accretion" scenario finds further support in numerical simulations, where mass accretes from the envelope onto the disk until the disk becomes unstable and unloads onto the star in a short burst of high accretion (Vorobyov \& Basu 2005, 2006, 2009, 2010; Zhu et al. 2009, 2010). Based on kinematic observations, the Class 0 protostar NGC1333 IRAS4A appears to be in a state of mass loading (Mottram et al. 2013).

\footnotetext{
${ }^{3} T_{\mathrm{bol}}$ is the temperature of a blackbody with the same mean frequency as the full spectral energy distribution (SED) from optical to millimeter wavelengths (Myers \& Ladd 1993). It generally increases as a protostar evolves (Chen et al. 1995). $L_{\mathrm{bol}}$ is the integrated flux across the SED; for embedded protostars, it is essentially equal to $L_{*}$.
} 


\subsection{Chemical tracers of episodic accretion}

Because of the difficulty of directly observing embedded accretion bursts, some groups are looking into the use of chemistry and molecular spectroscopy as an alternative tracer. Each accretion burst causes an increase in luminosity, which heats up the entire circumstellar envelope. The dust temperature responds particularly fast to a change in luminosity: $<100 \mathrm{~s}$ at $10 \mathrm{AU}$ away from the star up to $5 \times 10^{6} \mathrm{~s}$ at a typical outer radius of $10^{4} \mathrm{AU}$ (Johnstone et al. 2013). The gas heats up more slowly and reaches a new equilibrium temperature after $\sim 10^{5} \mathrm{~s}$ at $10 \mathrm{AU}$ and $\sim 10^{9} \mathrm{~s}$ or $\sim 30$ $\mathrm{yr}$ at $10^{4} \mathrm{AU}$. That last value is comparable to the expected duration of $\sim 100 \mathrm{yr}$ for the strongest accretion bursts (Vorobyov \& Basu 2005; Dunham \& Vorobyov 2012).

The relevant chemistry is driven by freeze-out and evaporation. When a neutral gas-phase molecules hits a dust grain, it sticks to the surface if the temperature $T$ is below some critical value called the evaporation temperature. This value varies from one molecule to the next; in a protostellar envelope, $T_{\mathrm{ev}} \approx 20 \mathrm{~K}$ for $\mathrm{CO}$ and $100 \mathrm{~K}$ for $\mathrm{H}_{2} \mathrm{O}$ (Fraser et al. 2001; Bisschop et al. 2006). The molecules sticking to the grain surface form an ice mantle. If a cold dust grain is heated up, the ices sublimate back into the gas according to their respective evaporation temperatures.

When the envelope heats up during an accretion burst, the evaporation timescale is less than a year wherever the new temperature exceeds $T_{\mathrm{ev}}$ for some molecule (Hasegawa et al. 1992; Visser \& Bergin 2012). When the burst ends and the envelope cools back down, the freeze-out timescale is much longer: $10^{4} \mathrm{yr}$ at a typical gas density of $10^{6} \mathrm{~cm}^{-3}$. This results in a prolonged period during which the gas and dust temperatures in the envelope are characteristic of a quiescent state of accretion, while the chemistry is still representative of the hot phase from the most recent burst. In other words, the quiescent phase features abundance patterns inconsistent with its low luminosity.

Commonly observed species like $\mathrm{CO}, \mathrm{HCO}^{+}$, and $\mathrm{N}_{2} \mathrm{H}^{+}$are potential tracers of episodic accretion, because their abundances are sensitive to changes in temperature (Lee 2007; Visser \& Bergin 2012; Vorobyov et al. 2013). For example, the chemistry of $\mathrm{HCO}^{+}$can be boiled down to the set of three reversible reactions shown on the right. The dominant formation channel is the reaction between $\mathrm{CO}$ and $\mathrm{H}_{3}^{+}$, which gets suppressed below $20 \mathrm{~K}$ due to freeze-out of $\mathrm{CO}$. Above $100 \mathrm{~K}, \mathrm{HCO}^{+}$is destroyed mainly by $\mathrm{H}_{2} \mathrm{O}$. At lower

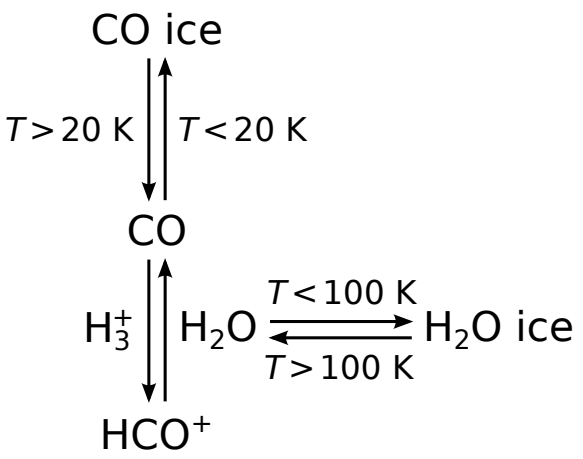
temperatures, $\mathrm{H}_{2} \mathrm{O}$ is frozen out and electrons become the main destruction channel (not shown).

Figure 5 shows the schematic radial abundance profile for $\mathrm{HCO}^{+}$before, during, and after a burst, based on the chemical models of Visser \& Bergin (in prep.). Before the burst, the profile breaks down into three components: a low inner abundance due to destruction by $\mathrm{H}_{2} \mathrm{O}$ above 100 $\mathrm{K}$; a high abundance between 20 and $100 \mathrm{~K}$, where $\mathrm{CO}$ is in the gas phase and $\mathrm{H}_{2} \mathrm{O}$ is not; and a lower outer abundance because $\mathrm{CO}$ is depleted onto the grains. ${ }^{4}$ The higher luminosity during the burst heats up the envelope and moves the $\mathrm{H}_{2} \mathrm{O}$ and $\mathrm{CO}$ evaporation radii outwards. The envelope quickly cools down after the burst (Johnstone et al. 2013), but the chemistry can only reset itself

\footnotetext{
${ }^{4}$ Due to the density gradient in the envelope, the freeze-out timescale of CO increases towards the outer edge. Hence, the $\mathrm{CO}$ and $\mathrm{HCO}^{+}$abundance both increase with radius in the part of the envelope below $20 \mathrm{~K}$.
} 

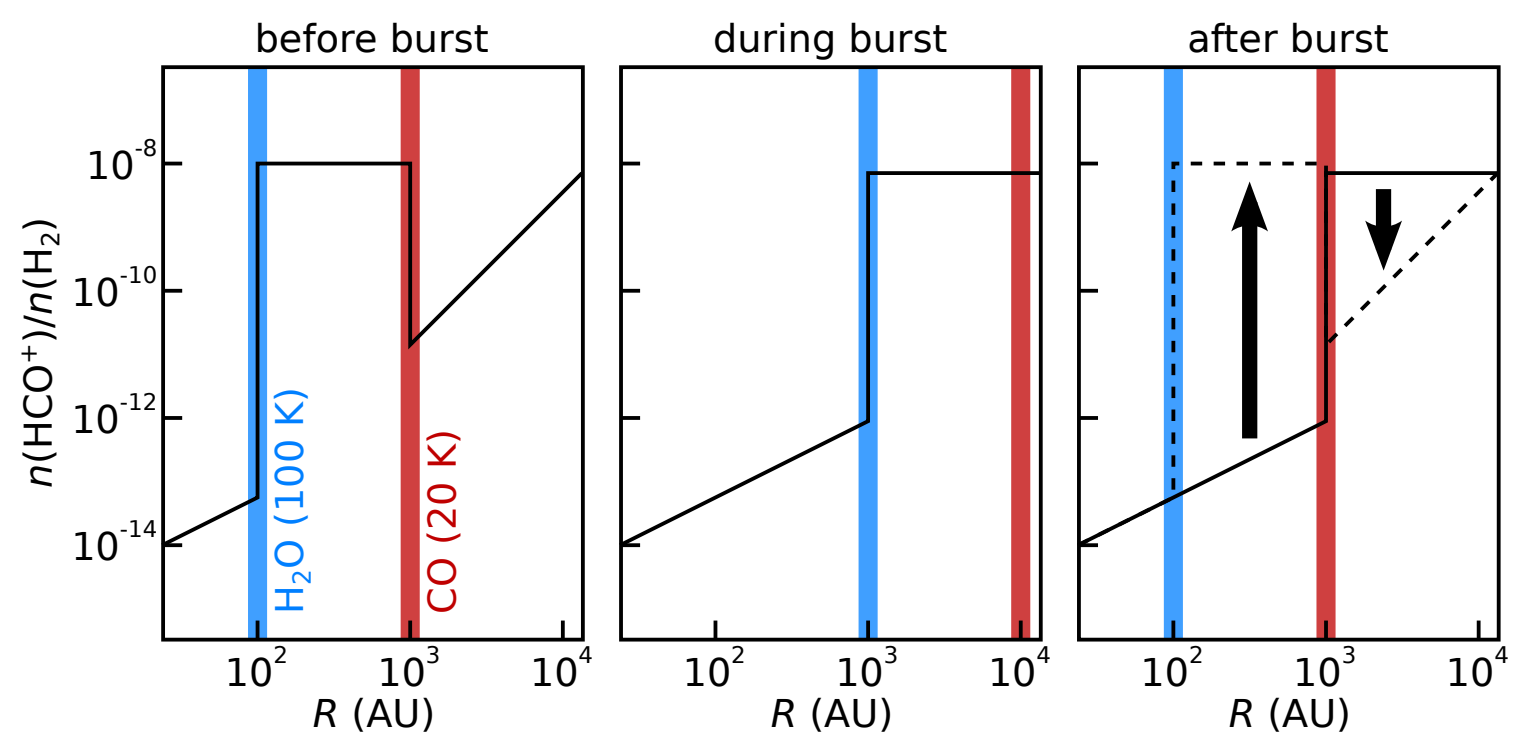

Figure 5: Schematic radial abundance profiles for $\mathrm{HCO}^{+}$in a protostellar envelope before, during, and after an accretion burst. The radii corresponding to the evaporation temperatures of $\mathrm{H}_{2} \mathrm{O}$ ice $(100 \mathrm{~K})$ and $\mathrm{CO}$ ice $(20 \mathrm{~K})$ are marked with blue and red bars. Based on Visser \& Bergin (in prep.).

according to the much longer timescales associated with freeze-out at different radii (Visser \& Bergin 2012). Until that happens, there is an excess of cold $\mathrm{HCO}^{+}(<20 \mathrm{~K})$ and a lack of warm $\mathrm{HCO}^{+}(20-100 \mathrm{~K})$ relative to the current dust temperature profile.

One way to observe such an anomalous $\mathrm{HCO}^{+}$abundance profile would be through singledish spectra of a low- and a high-excitation line, such as $J=3-2$ and 7-6 (Visser \& Bergin in prep.). Spatially resolved interferometric observations offer even better constraints. For example, Jørgensen et al. (2013) targeted the $J=4-3$ line of the optically thin isotopolog $\mathrm{H}^{13} \mathrm{CO}^{+}$in the Class 0 protostar IRAS 15398 with the Atacama Large Millimeter Array (ALMA). The emission shows up as a ring around the central star. The dust temperature inferred at the inner edge of the ring is only $30 \mathrm{~K}$, too low for $\mathrm{H}_{2} \mathrm{O}$ to be abundant in the gas and destroy $\mathrm{HCO}^{+}$. Hence, it appears the temperature was higher in the past and the chemistry is still in the process of readjusting to the current cold environment. The temperature discrepancy suggests a burst that raised the stellar luminosity by a factor of 100 . Based on the freeze-out timescale of $\mathrm{H}_{2} \mathrm{O}$, this burst happened 100-1000 yr ago (Jørgensen et al. 2013).

Other chemical evidence for episodic accretion comes in the form of Spitzer observations of the $\mathrm{CO}_{2}$ ice band at $15 \mu \mathrm{m}$. The shape and width of this spectral feature depend on whether the $\mathrm{CO}_{2}$ ice is pure or mixed with $\mathrm{CO}$ and/or $\mathrm{H}_{2} \mathrm{O}$ (Ehrenfreund et al. 1997; van Broekhuizen et al. 2006). In protostellar envelopes, $\mathrm{CO}_{2}$ ice forms out of $\mathrm{CO}$ ice below $20 \mathrm{~K}$. Pure $\mathrm{CO}_{2}$ ice - known by a characteristic double peak in the $15-\mu \mathrm{m}$ band - is produced by heating the grains to 20 $40 \mathrm{~K}$ to evaporate $\mathrm{CO} . \mathrm{CO}_{2}$ is more tightly bound and remains frozen. This purification step is irreversible (Hagen et al. 1983), so if pure $\mathrm{CO}_{2}$ ice is detected below $20 \mathrm{~K}$, it is an indication of a warmer past (Lee 2007). In embedded protostars with luminosities of less than $1 L_{\odot}$, the bulk of the envelope is too cold to form pure $\mathrm{CO}_{2}$ ice at detectable levels. Nonetheless, Kim et al. $(2011,2012)$ detected a clear double-peaked line profile in the $\mathrm{CO}_{2}$ ice band for six out of 19 
such low-luminosity sources, and concluded that they must have experienced an accretion burst at some point. Unlike the $\mathrm{H}^{13} \mathrm{CO}^{+}$data from Jørgensen et al. (2013), the $\mathrm{CO}_{2}$ ice bands offer no information about when these bursts occurred.

\section{Conclusions}

Despite decades of work, many aspects of low-mass star formation remain poorly understood. Recent observations with Spitzer and Herschel have shed new light on the energy balance in embedded protostars and on the relative importance of feedback from UV photons and shocks. Another topic of current interest is episodic accretion, i.e., a scenario in which a young star gains most of its mass in short bursts while spending most of the time in a state of low accretion. Chemistry and molecular spectroscopy are proving to be invaluable tools in the worldwide efforts to characterize and understand energetic feedback and episodic accretion in more detail.

\section{References}

Adams, F. C., \& Shu, F. H. 1985, ApJ, 296, 655

Alexander, R., Pascucci, I., Andrews, S., Armitage, P., \& Cieza, L. 2014, Protostars and Planets VI (arXiv: 1311.1819)

André, P., Di Francesco, J., Ward-Thompson, D., et al. 2014, Protostars and Planets VI (arXiv: 1312.6232)

André, P., Ward-Thompson, D., \& Barsony, M. 1993, ApJ, 406, 122

André, P., Ward-Thompson, D., \& Barsony, M. 2000, Protostars and Planets IV, 59

Andrews, S. M., \& Williams, J. P. 2007, ApJ, 671, 1800

Arce, H. G., Shepherd, D., Gueth, F., et al. 2007, Protostars and Planets V, 245

Arthur, S. J. 2008, in IAU Symp. 250: Massive Stars as Cosmic Engines, ed. F. Bresolin, P. A. Crowther, \& J. Puls, 355-360

Audard, M., Ábrahám, P., Dunham, M. M., et al. 2014, Protostars and Planets VI (arXiv: 1401.3368)

Bally, J., Reipurth, B., \& Davis, C. J. 2007, Protostars and Planets V, 215

Bate, M. R. 2012, MNRAS, 419, 3115

Benz, W., Ida, S., Alibert, Y., Lin, D., \& Mordasini, C. 2014, Protostars and Planets VI

Bergin, E. A., \& Tafalla, M. 2007, ARA\&A, 45, 339

Bertout, C. 1989, ARA\&A, 27, 351

Bisschop, S. E., Fraser, H. J., Öberg, K. I., van Dishoeck, E. F., \& Schlemmer, S. 2006, A\&A, 449, 1297

Cameron, A. G. W. 1988, ARA\&A, 26, 441

Ceccarelli, C., Caselli, P., Bockelee-Morvan, D., et al. 2014, Protostars and Planets VI

Chen, H., Myers, P. C., Ladd, E. F., \& Wood, D. O. S. 1995, ApJ, 445, 377

Chu, Y.-H. 2008, in IAU Symp. 250: Massive Stars as Cosmic Engines, ed. F. Bresolin, P. A. Crowther, \& J. Puls, 341-354

de Graauw, T., Helmich, F. P., Phillips, T. G., et al. 2010, A\&A, 518, L6

Dionatos, O., Jørgensen, J. K., Green, J. D., et al. 2013, A\&A, 558, A88 
Dobbs, C. L., Krumholz, M. R., Ballesteros-Paredes, J., et al. 2014, Protostars and Planets VI (arXiv: 1312.3223)

Dopita, M. A. 2008, in IAU Symp. 250: Massive Stars as Cosmic Engines, ed. F. Bresolin, P. A. Crowther, \& J. Puls, 367-378

Dullemond, C. P., Hollenbach, D., Kamp, I., \& D’Alessio, P. 2007, Protostars and Planets V, 555

Dunham, M. M., Crapsi, A., Evans, II, N. J., et al. 2008, ApJS, 179, 249

Dunham, M. M., \& Vorobyov, E. I. 2012, ApJ, 747, 52

Dunham, M. M., Stutz, A. M., Allen, L. E., et al. 2014, Protostars and Planets VI (arXiv: 1401.1809)

Ehrenfreund, P., Boogert, A. C. A., Gerakines, P. A., Tielens, A. G. G. M., \& van Dishoeck, E. F. 1997, A\&A, 328, 649

Enoch, M. L., Evans, II, N. J., Sargent, A. I., \& Glenn, J. 2009, ApJ, 692, 973

Espaillat, C., Andrews, S., Calvet, N., et al. 2014, Protostars and Planets VI

Evans, N. J., Dunham, M. M., Jørgensen, J. K., et al. 2009, ApJS, 181, 321

Fischer, W. J., Megeath, S. T., Stutz, A. M., et al. 2013, Astron. Nachr., 334, 53

Frank, A., Cabrit, S., Ray, T., et al. 2014, Protostars and Planets VI

Fraser, H. J., Collings, M. P., McCoustra, M. R. S., \& Williams, D. A. 2001, MNRAS, 327, 1165

Giannini, T., Nisini, B., \& Lorenzetti, D. 2001, ApJ, 555, 40

Goicoechea, J. R., Cernicharo, J., Karska, A., et al. 2012, A\&A, 548, A77

Goldsmith, P. F., \& Langer, W. D. 1999, ApJ, 517, 209

Green, J. D., Evans, II, N. J., Jørgensen, J. K., et al. 2013, ApJ, 770, 123

Hagen, W., Tielens, A. G. G. M., \& Greenberg, J. M. 1983, A\&AS, 51, 389

Hartmann, L., \& Kenyon, S. J. 1996, ARA\&A, 34, 207

Hasegawa, T. I., Herbst, E., \& Leung, C. M. 1992, ApJS, 82, 167

Helled, R., Bodenheimer, P., Podolak, M., et al. 2014, Protostars and Planets VI (arXiv: 1311.1142)

Herbig, G. H. 1977, ApJ, 217, 693

Herczeg, G. J., Karska, A., Bruderer, S., et al. 2012, A\&A, 540, A84

Hogerheijde, M. R., van Dishoeck, E. F., Blake, G. A., \& van Langevelde, H. J. 1998, ApJ, 502, 315

Johansen, A., Blum, J., Tanaka, H., et al. 2014, Protostars and Planets VI (arXiv: 1402.1344)

Johnstone, D., Hendricks, B., Herczeg, G. J., \& Bruderer, S. 2013, ApJ, 765, 133

Jørgensen, J. K., Bourke, T. L., Myers, P. C., et al. 2007, ApJ, 659, 479

Jørgensen, J. K., Visser, R., Sakai, N., et al. 2013, ApJ, 779, L22

Kant, I. 1755, Allgemeine Naturgeschichte und Theorie des Himmels (Königsberg \& Leipzig: J.F. Petersen)

Karska, A., Herczeg, G. J., van Dishoeck, E. F., et al. 2013, A\&A, 552, A141

Kennicutt, R. C., \& Evans, N. J. 2012, ARA\&A, 50, 531

Kenyon, S. J., Gomez, M., Marzke, R. O., \& Hartmann, L. 1994, AJ, 108, 251

Kenyon, S. J., \& Hartmann, L. 1995, ApJS, 101, 117

Kenyon, S. J., Hartmann, L. W., Strom, K. M., \& Strom, S. E. 1990, AJ, 99, 869

Kessler, M. F., Steinz, J. A., Anderegg, M. E., et al. 1996, A\&A, 315, L27

Kim, H. J., Evans, II, N. J., Dunham, M. M., Lee, J.-E., \& Pontoppidan, K. M. 2012, ApJ, 758, 38

Kim, H. J., Evans, II, N. J., Dunham, M. M., et al. 2011, ApJ, 729, 84 
Klein, R. I., Inutsuka, S.-I., Padoan, P., \& Tomisaka, K. 2007, Protostars and Planets V, 99

Kristensen, L. E., van Dishoeck, E. F., Benz, A. O., et al. 2013, A\&A, 557, A23

Lada, C. J. 1999, in The Origin of Stars and Planetary Systems, ed. C. J. Lada \& N. D. Kylafis (Dordrecht: Kluwer), 143

Laplace, P.-S. 1796, Exposition du Système du Monde (Paris: Cercle-Social)

Lee, J., Lee, J.-E., Lee, S., et al. 2013, ApJS, 209, 4

Lee, J.-E. 2007, J. Korean Astron. Soc., 40, 83

Li, Z.-Y., Banerjee, R., Pudritz, R. E., et al. 2014, Protostars and Planets VI (arXiv: 1401.2219)

Lindberg, J. E., Jørgensen, J. K., Green, J. D., et al. 2014, A\&A, in press (arXiv: 1311.7657)

Manoj, P., Watson, D. M., Neufeld, D. A., et al. 2013, ApJ, 763, 83

Matthews, B. C., Krivov, A. V., Wyatt, M. C., Bryden, G., \& Eiroa, C. 2014, Protostars and Planets VI (arXiv: 1401.0743)

McKee, C. F., \& Ostriker, E. C. 2007, ARA\&A, 45, 565

Mottram, J. C., van Dishoeck, E. F., Schmalzl, M., et al. 2013, A\&A, 558, A126

Murillo, N. M., Lai, S.-P., Bruderer, S., Harsono, D., \& van Dishoeck, E. F. 2013, A\&A, 560, A103

Myers, P. C., Adams, F. C., Chen, H., \& Schaff, E. 1998, ApJ, 492, 703

Myers, P. C., \& Ladd, E. F. 1993, ApJ, 413, L47

Neufeld, D. A. 2012, ApJ, 749, 125

Neufeld, D. A., Nisini, B., Giannini, T., et al. 2009, ApJ, 706, 170

Nisini, B., Giannini, T., \& Lorenzetti, D. 2002, ApJ, 574, 246

Oey, M. S., Voges, E. S., Walterbos, R. A. M., et al. 2008, in IAU Symp. 250: Massive Stars as Cosmic Engines, ed. F. Bresolin, P. A. Crowther, \& J. Puls, 385-390

Offner, S. S. R., Kratter, K. M., Matzner, C. D., Krumholz, M. R., \& Klein, R. I. 2010, ApJ, 725, 1485

Offner, S. S. R., \& McKee, C. F. 2011, ApJ, 736, 53

Pilbratt, G. L., Riedinger, J. R., Passvogel, T., et al. 2010, A\&A, 518, L1

Poglitsch, A., Waelkens, C., Geis, N., et al. 2010, A\&A, 518, L2

Raga, A. C., Velázquez, P. F., Cantó, J., \& Masciadri, E. 2002, A\&A, 395, 647

Ray, T., Dougados, C., Bacciotti, F., Eislöffel, J., \& Chrysostomou, A. 2007, Protostars and Planets $\mathrm{V}, 231$

Raymond, S. N., Kokubo, E., Morbidelli, A., Morishima, R., \& Walsh, K. J. 2014, Protostars and Planets VI (arXiv: 1312.1689)

Reipurth, B. 1989, Nature, 340, 42

Robitaille, T. P., Whitney, B. A., Indebetouw, R., Wood, K., \& Denzmore, P. 2006, ApJS, 167, 256

Scholz, A., Froebrich, D., \& Wood, K. 2013, MNRAS, 430, 2910

Shang, H., Li, Z.-Y., \& Hirano, N. 2007, Protostars and Planets V, 261

Shu, F. H. 1977, ApJ, 214, 488

Shu, F. H., Adams, F. C., \& Lizano, S. 1987, ARA\&A, 25, 23

Shu, F. H., Li, Z.-Y., \& Allen, A. 2004, in ASP Conf. Ser. 323: Star Formation in the Interstellar Medium: In Honor of David Hollenbach, ed. D. Johnstone, F. C. Adams, D. N. C. Lin, D. A. Neufeld, \& E. C. Ostriker (San Francisco: ASP), 37

Spaans, M., Hogerheijde, M. R., Mundy, L. G., \& van Dishoeck, E. F. 1995, ApJ, 455, L167

Stahler, S. W. 1983, ApJ, 274, 822 
Swedenborg, E. 1734, Opera Philosophica et Mineralia (Dresden \& Leipzig: F. Hekel)

Takakuwa, S., Saito, M., Lim, J., et al. 2012, ApJ, 754, 52

Tobin, J. J., Hartmann, L., Chiang, H.-F., et al. 2012, Nature, 492, 83

van Broekhuizen, F. A., Groot, I. M. N., Fraser, H. J., van Dishoeck, E. F., \& Schlemmer, S. 2006,

A\&A, 451, 723

van Dishoeck, E. F. 2004, ARA\&A, 42, 119

van Dishoeck, E. F., Bergin, E. A., Lis, D. C., \& Lunine, J. I. 2014, Protostars and Planets VI (arXiv: 1401.8103)

van Dishoeck, E. F., \& Blake, G. A. 1998, ARA\&A, 36, 317

van Dishoeck, E. F., Blake, G. A., Draine, B. T., \& Lunine, J. I. 1993, Protostars and Planets III, 163

van Kempen, T. A., van Dishoeck, E. F., Güsten, R., et al. 2009, A\&A, 501, 633

van Kempen, T. A., Green, J. D., Evans, N. J., et al. 2010a, A\&A, 518, L128

van Kempen, T. A., Kristensen, L. E., Herczeg, G. J., et al. 2010b, A\&A, 518, L121

Visser, R., \& Bergin, E. A. 2012, ApJ, 754, L18

Visser, R., Kristensen, L. E., Bruderer, S., et al. 2012, A\&A, 537, A55

Vorobyov, E. I., Baraffe, I., Harries, T., \& Chabrier, G. 2013, A\&A, 557, A35

Vorobyov, E. I., \& Basu, S. 2005, ApJ, 633, L137

Vorobyov, E. I., \& Basu, S. 2006, ApJ, 650, 956

Vorobyov, E. I., \& Basu, S. 2009, MNRAS, 393, 822

Vorobyov, E. I., \& Basu, S. 2010, ApJ, 719, 1896

Watson, D. M., Bohac, C. J., Hull, C., et al. 2007, Nature, 448, 1026

Werner, M. W., Roellig, T. L., Low, F. J., et al. 2004, ApJS, 154, 1

White, R. J., Greene, T. P., Doppmann, G. W., Covey, K. R., \& Hillenbrand, L. A. 2007, Protostars and Planets V, 117

Y1ldı, U. A., Kristensen, L. E., van Dishoeck, E. F., et al. 2012, A\&A, 542, A86

Yıldı, U. A., Kristensen, L. E., van Dishoeck, E. F., et al. 2013, A\&A, 556, A89

Zhu, Z., Hartmann, L., \& Gammie, C. 2009, ApJ, 694, 1045

Zhu, Z., Hartmann, L., \& Gammie, C. 2010, ApJ, 713, 1143 